\title{
Harmonic clipping contours: numerical computation and extension to higher harmonics
}

\author{
Roberto Quaglia, Steve Cripps \\ School of Engineering \\ Cardiff University \\ 14-17 The Parade, Cardiff, CF24 3AA \\ Email: quagliar@cardiff.ac.uk
}

\begin{abstract}
This paper presents the computation of harmonic clipping contours using a Matlab script that guarantees a fast calculation. Thanks to the numerical nature of the solution, an arbitrary number of harmonics can be taken into account with minimal added computational cost. The results for second harmonic clipping contours are compared with the analytical solutions available in literature, while for the first time $3^{\text {rd }}$ harmonics clipping contours are presented. The latter allow the improvement of the output power and the efficiency achievable with the active device, while strong-linearity due to clipping can be consciously avoided in the design stage.
\end{abstract}

\section{INTRODUCTION}

The power amplifier (PA) is one of the most critical components in radio frequency (RF) transmitters due to its strong impact on linearity, power efficiency, and overall cost. Designers can rely on computer-aided design, exploiting large signal models of the active devices to finalize the schematic of the PA, accounting for all the given constraints. However, it is common practice, in the first stages of the design, to adopt a simplified approach based on the identification of the load terminations to be applied at the device ports. For narrowband design, tuned-load class $\mathrm{AB}$, class $\mathrm{F}$, or second harmonic tuned PAs refer to precise harmonic load locations, that are derived from theoretical analysis [1], [2] and chosen according to the technology of the device, the frequency of operation, and the feasibility of the matching networks. When turning to wideband design, as required by last-generation communication systems, it becomes difficult to guarantee a fixed load termination over a large bandwidth. To mitigate this issue, a feasible approach is to rely on the continuous PA modes, revealed in [3], and extended in [4], [5] to other classes of PAs. A limitation of the continuous modes's theory is represented by the requirement of a purely reactive impedance at harmonic frequencies, that in practical realization of matching networks is hardly achieved. This aspect is even more evident in broadband PAs, where the harmonics of the lower band fundamentals are very close, or even inside, the higher portion of the fundamental band. Moreover, a clear indication of the effect on output power and linearity of dissipative higher harmonics was not available, and empirical or a posteriori methods have been used to verify this effect [6]. The clipping contours have been first introduced in [7], and then in [8] with a different formulation, to address the presence of second harmonic loads containing a resistance. For a given second harmonic load, the clipping contours identify a region of fundamental impedance loads, i.e., the contour, that lead to a zero-grazing voltage waveform. The contour divides the Smith Chart in two regions: one on the left of the contour, where loads lead to a waveform non-reaching zero, and on the right, where the loads lead to a clipped voltage waveform. Alternatively, if the fundamental load is fixed, the second harmonic clipping contour can be plotted. The clipping contours are a powerful method to understand the involved trade-offs when designing the matching network. In fact, it can be seen that reducing the fundamental voltage, i.e., reducing the output power, the region of second harmonic loads that avoid clipping is enhanced, thus relaxing the necessity of a purely reactive second harmonic load [7]. In this paper, a numerical evaluation of the clipping contours is presented, allowing for the first time to account for the effect of higher harmonics, that were considered at short circuit in [7], [8]. The plot of the contours is performed in Matlab exploiting a computationally efficient procedure. Experimental characterization is ongoing to prove the advantages deriving from higher harmonics inclusion.

\section{COMPUTATION OF CLIPPING CONTOURS}

The adopted approach assumes that the intrinsic device plane is considered, see Fig. 1, and that the drain current waveform is known. Moreover, zero voltage is considered as the limit for clipping, but all the calculations can be re-scaled to account for a higher knee voltage. The periodical drain current waveform, if characterised by even symmetry, can be written as a Fourier series with cosine only terms:

$$
i_{\mathrm{ds}}(\theta)=I_{0}+\sum_{n=1}^{\infty} I_{n} \cos (n \theta)
$$

where $\theta=2 \pi f_{0} t$, with $f_{0}$ fundamental frequency, $I_{0}$ is the DC current component, and $I_{n}$ are the current harmonic components. According to Ohm's law, and considering har- 


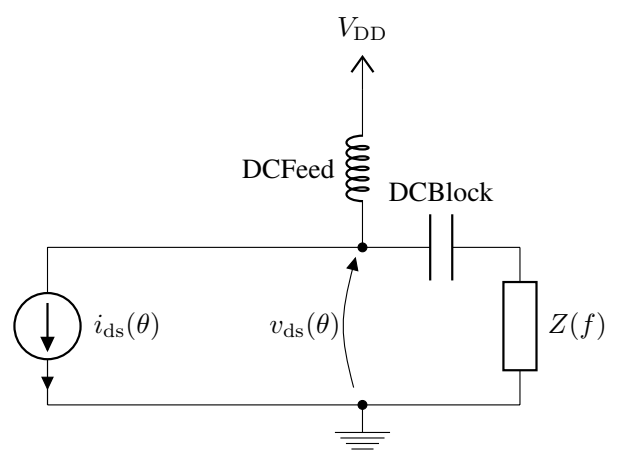

Fig. 1. Simplified schematic used for the analysis.

monics higher than $N$ as short circuits, the voltage waveform becomes:

$$
\begin{aligned}
& v_{\mathrm{ds}}(\theta)= \\
& =V_{\mathrm{DD}}+\sum_{n=1}^{N}\left(\Re e\left\{V_{n}\right\} \cos (n \theta)+\Im m\left\{V_{n}\right\} \sin (n \theta)\right) \\
& =V_{\mathrm{DD}}-\sum_{n=1}^{N}\left(R_{n} I_{n} \cos (n \theta)+X_{n} I_{n} \sin (n \theta)\right)
\end{aligned}
$$

where $Z_{n}=R_{n}+j X_{n}$ is the $n$-th harmonic impedance presented at the device port, $Z\left(n f_{0}\right)$, and $V_{\mathrm{DD}}$ is the drain bias voltage. If the current waveform is not even, current sin components are present as well, and care must be taken when evaluating the voltage waveform components. However, the proposed procedure is still valid.

The clipping contours at the $k$-th harmonic can be plotted if the $i$-th harmonic loads, with $i \neq k$, are known. To build the clipping contours, a grid of loads is created and assigned to $Z_{k}$. For each load, the voltage waveform is built according to (2) on a period $-\pi \leq \theta \leq \pi$, and its minimum $M_{\mathrm{v}}\left(R_{k}, X_{k}\right)$ is evaluated. The loads at which the minimum of the waveform is equal to zero represent the clipping contour.

Unfortunately, being the loads located on a discrete grid, it is highly improbable that the locus of $M_{\mathrm{v}}\left(R_{k}, X_{k}\right)=0$ can be exactly identified. For this reason, it has been decided to use the Matlab function contour, that given a function of twovariables quickly identifies the contour levels. In particular, the contour level at which $M_{\mathrm{v}}\left(R_{k}, X_{k}\right)=0$ is shown. The Matlab contour function still performs an interpolation, so it is necessary to use a large number of impedance points to achieve an accurate clipping contour. Moreover, also $\theta$ is discretized into a column vector with $T$ points, and its discretization step must be low enough for an accurate identification of the minimum. As a consequence, to avoid a non-acceptable computational time for the contour generation, the algorithm has been designed exploiting the capability of Matlab to efficiently operate on vectors and matrices.

The impedance $Z_{k}$ is represented by a $P \times M$ matrix $\tilde{Z}_{k}$, obtained by inner product of a column vector of length $P$ containing the complex numbers of unitary module and phase equal to all the possible phase of $Z_{k}$, and a row vector with length $M$ containing all the possible modulus of $Z_{k}$. An important observation is made to limit the range of $\left|Z_{k}\right|$ to $R_{\mathrm{m}} \leq\left|Z_{k}\right| \leq R_{\mathrm{M}}$. In fact, considering the limit case of all the $i$-th harmonics in phase or phase opposition at the negative peak of the $k$-th harmonic, the amplitude of the $k$-th harmonic voltage must be between $V_{\mathrm{m}}$ and $V_{\mathrm{M}}$ :

$$
\begin{aligned}
& V_{\mathrm{m}}=V_{\mathrm{DD}}-\sum_{i}\left|V_{i}\right| \\
& V_{\mathrm{M}}=V_{\mathrm{DD}}+\sum_{i}\left|V_{i}\right|
\end{aligned}
$$

to eventually achieve zero grazing. If $V_{\mathrm{m}}$ is negative, it is changed to zero. Once $V_{\mathrm{m}}$ and $V_{\mathrm{M}}$ are identified, the corresponding impedance limits $R_{\mathrm{m}}$ and $R_{\mathrm{M}}$ can be extracted, since the $I_{k}$ current is known. Only passive loads are considered, thus the phase of $Z_{k}$ is limited between $-\pi / 2$ and $\pi / 2$.

The matrix $\tilde{Z}_{k}$ is then reshaped columnwise into a row vector $Z_{k}$ with length $M P$, and the corresponding $V_{k}$ values are obtained as a scalar product between $Z_{k}$ and the constant $I_{k}$. This permits the calculation of $\tilde{v}_{\mathrm{ds}}$, the matrix of voltage waveforms that will have dimension $T \times M P$. Each column of $\tilde{v}_{\mathrm{ds}}$ is a voltage waveform corresponding to a load $Z_{k}$. By defining $\tilde{1}_{\mathrm{M}}$ as a matrix with dimension $T \times M P$, and $1_{\mathrm{V}}$ as a row vector with dimension $M P$, both composed by unitary elements, the waveform matrix can be obtained as:

$$
\begin{aligned}
& \tilde{v}_{\mathrm{ds}}=V_{\mathrm{DD}} \cdot \tilde{1}_{\mathrm{M}}+\cos (k \theta) \cdot \Re e\left\{V_{k}\right\}+\sin (k \theta) \cdot \Im m\left\{V_{k}\right\}+ \\
& +\sum_{i}\left(\Re e\left\{V_{i}\right\} \cos (i \theta) \cdot 1_{\mathrm{V}}+\Im m\left\{V_{i}\right\} \sin (i \theta) \cdot 1_{\mathrm{V}}\right) .
\end{aligned}
$$

Finally, the row vector $M_{\mathrm{V}}$, with length $M P$, is evaluated with the function $\min \left(\tilde{v}_{\mathrm{ds}}\right)$, that performs the columnwise minimum of $\tilde{v}_{\mathrm{ds}}$. The matrix $\tilde{M}_{\mathrm{V}}$, with dimension $P \times M$, is obtained reshaping $M_{\mathrm{V}}$.

The plot in the Smith Chart is then obtained simply mapping $\tilde{Z}_{k}$ into a matrix $\tilde{\Gamma}_{k}$ of reflection coefficients, using $R_{\text {opt }}$ as normalization impedance, i.e., the theoretical optimum load with shorted harmonic, $R_{\mathrm{opt}}=V_{\mathrm{DD}} / I_{1}$. The function $\operatorname{contour}\left(\Re\{\Gamma\}, \Im\{\Gamma\}, \tilde{Z}_{k}\right)$ is finally adopted, visualizing the zero value contour.

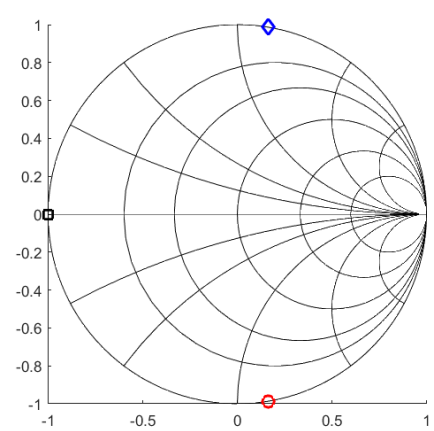

(a)

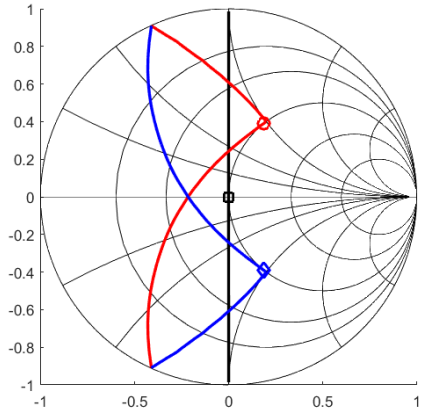

(b)
Fig. 2. Second harmonic loads (a) and fundamental clipping contour (b) for class B (black square), class $\mathbf{J}$ (red circle), and class $\mathrm{J}^{-1}$ (blue diamond). 
The algorithm is tested with simple loading conditions, as for example class $\mathrm{B}, \mathrm{J}$, and $\mathrm{J}^{-1}$, assuming a class $\mathrm{B}$ current waveform. Fig. 2 shows, for these 3 classes, the fixed second harmonic load on the lef, and the fundamental clipping contour, on the right, with the symbols placed at the maximum power loads. The computation time, using $T=361, M=$ $10, P=37$, and an Intel Core i7 $2.6 \mathrm{GHz}$ processor, is around $0.33 \mathrm{~s}$ when starting from an empty workspace, while it decreases to $\approx 0.05 \mathrm{~s}$ if the variables are already initiated.

\section{Clipping CONTOURS WITH $2^{\text {nd }}$ HARMONIC}

Before proceeding to insert higher harmonics, the algorithm is also tested against the results provided in [7]. Fig. 3 shows how the fundamental clipping contours (right) are affected by a second harmonic load (left) moving from short circuit to a purely resistive impedance, again assuming a class $\mathrm{B}$ waveform. It is clear that, for an increasing second harmonic load, the fundamental load must be moved towards lower impedances to avoid clipping, meaning a reduction of output power and efficiency. Similarly, but providing arbitrary second

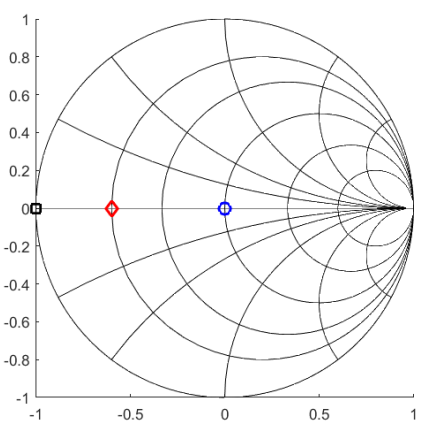

(a)

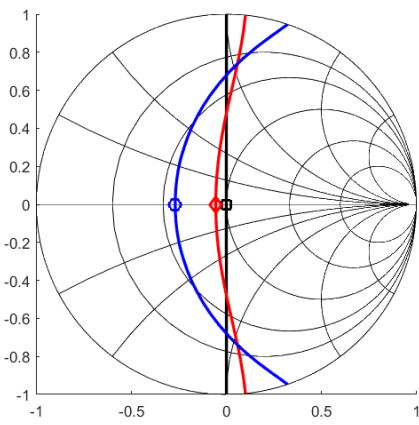

(b)
Fig. 3. Second harmonic loads (a) and fundamental clipping contour (b) for 3 different cases. $Z_{2}=0$ (black square), $Z_{2}=R_{\mathrm{opt}} / 4$ (red diamond), and $Z_{2}=R_{\text {opt }}$ (blue circle).

harmonic complex load, different clipping contours can be seen in Fig. 4.

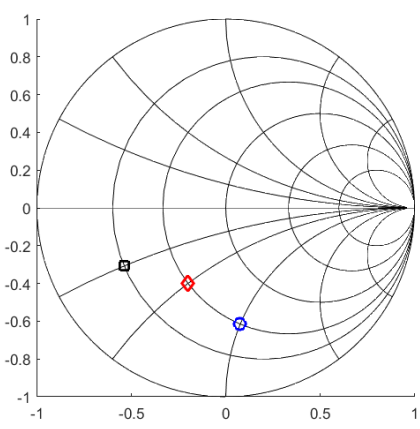

(a)

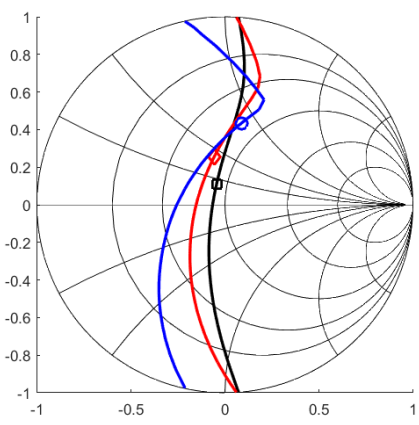

(b)
Fig. 4. Second harmonic loads (a) and fundamental clipping contour (b) for 3 different cases. $Z_{2}=(1-j) R_{\mathrm{opt}} / 4$ (black square), $Z_{2}=(1-j) R_{\mathrm{opt}} / 2$ (red diamond), and $Z_{2}=(1-j 2) R_{\mathrm{opt}} / 2$ (blue circle).
A situation of great interest is also when the fundamental load is fixed, i.e., a certain power reduction is accepted, and the second harmonic clipping contour needs to be found. Fig. 5 reports 3 cases, one with the fundamental load (left) at its power optimum, and the other two with reduction of 0.95 and 0.9 , respectively. The second harmonic clipping contour (right) passes from the short circuit only, to a much larger impedance region, allowing for more flexibility in the matching network design without clipping the waveform. The symbols on the

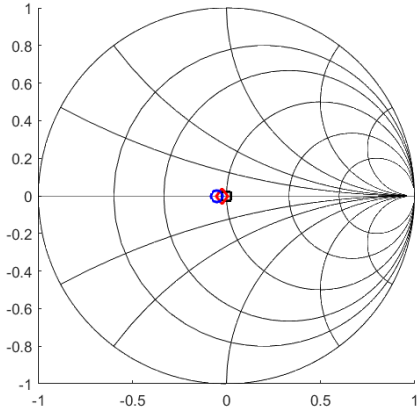

(a)

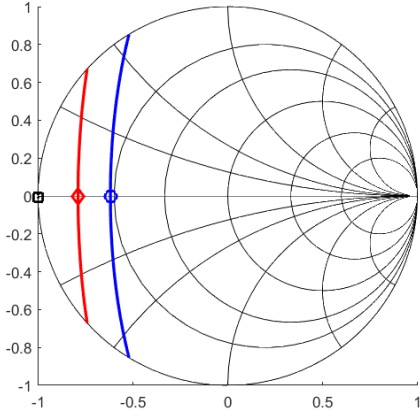

(b)
Fig. 5. Fundamental loads (a) and second harmonic clipping contour (b) for 3 different cases. Class B (black square), $Z_{1}=0.95 R_{\mathrm{opt}}$ (red diamond), and $Z_{1}=0.9 R_{\text {opt }}$ (blue circle).

second harmonic clipping contours are only shown as markers; in fact, they do not indicate the locus of maximum power, being the latter determined by the fundamental load.

\section{Clipping CONTOURS With $3^{\text {rd }}$ HARMONiC}

The insertion of high harmonics, and in particular the third harmonic, in the voltage waveform can provide beneficial effects in terms of power and efficiency: class $\mathrm{F}$ is an example of this application. For this reason, it is of great interest to observe the clipping contours including the effect of the third harmonic. A $10 \%$ class $\mathrm{AB}$ bias is used instead of class B to

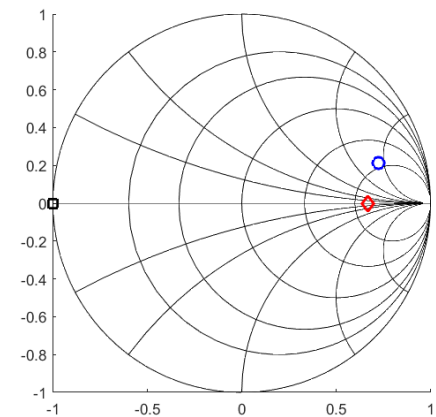

(a)

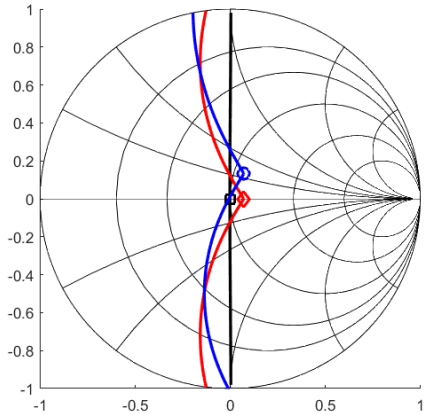

(b)
Fig. 6. Third harmonic loads (a) and fundamental clipping contours (b) for 3 different cases, fixing $Z_{2}=0 . Z_{3}=0$ (black square), $Z_{3}=5 R_{\mathrm{opt}}$ (red diamond), and $Z_{3}=(5+j 5) R_{\text {opt }}$ (blue circle).

generate a finite third harmonic current. As a first exercise, 
the fundamental clipping contours with short circuited second harmonic and different values of third harmonic are calculated, and reported in Fig. 6. The fundamental load, when applying a high third harmonic load, is allowed to reach a higher impedance than the optimum for tuned load. This leads to an output power $\approx 1.16$ larger than the correspondent tuned load, consistently with the class F theory results. Moreover, the adoption of a complex third harmonic load moves the fundamental optimum load away from the real impedance axis. Fig. 7 reports the normalized voltage waveforms for the three

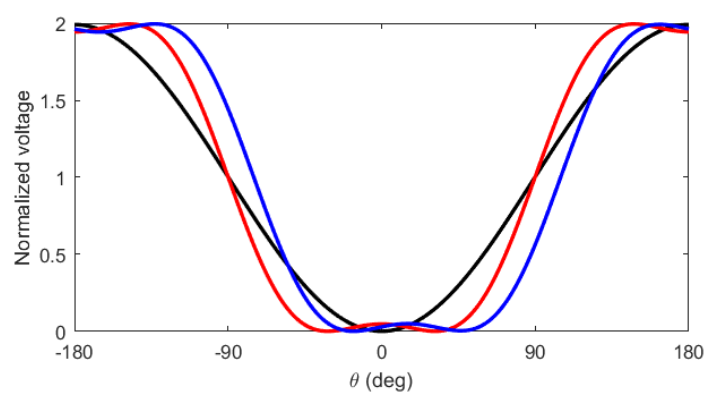

Fig. 7. Normalized voltage waveform for 3 different cases, fixing $Z_{2}=0$. $Z_{3}=0$ (black), $Z_{3}=5 R_{\text {opt }}$ (red), and $Z_{3}=(5+j 5) R_{\text {opt }} / \sqrt{2}$ (blue).

presented cases, selecting the maximum output power load at fundamental. The class F-like waveform is clearly visible, and the change of phase of third harmonic load results in a shift of the full waveform.

A more realistic case is represented by dissipative second and third harmonics. In particular, in many continuous mode implementations the second harmonic is usually in the third quadrant of the Smith Chart, while the third harmonic tends to rotate towards the second and first quadrants. Fig. 8 shows

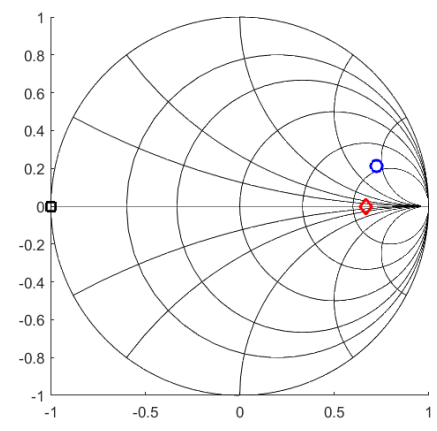

(a)

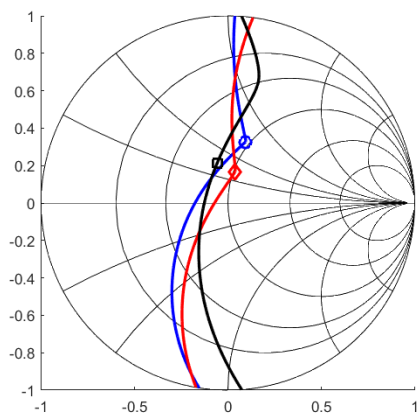

(b)
Fig. 8. Third harmonic loads (a) and fundamental clipping contours (b) for 3 different cases, fixing $Z_{2}=(1-j) R_{\mathrm{opt}} / 2 . Z_{3}=0$ (black square), $Z_{3}=5 R_{\text {opt }}$ (red diamond), and $Z_{3}=(5+j 5) R_{\text {opt }} / \sqrt{2}$ (blue circle).

the fundamental clipping contours when the second harmonic is $Z_{2}=(1-j) R_{\text {opt }} / 2$, for the three different third harmonic loads. Introducing a high impedance for the third harmonic has a beneficial effect, and the maximum output power is back to the tuned load value. Fig. 9 reports the normalized voltage

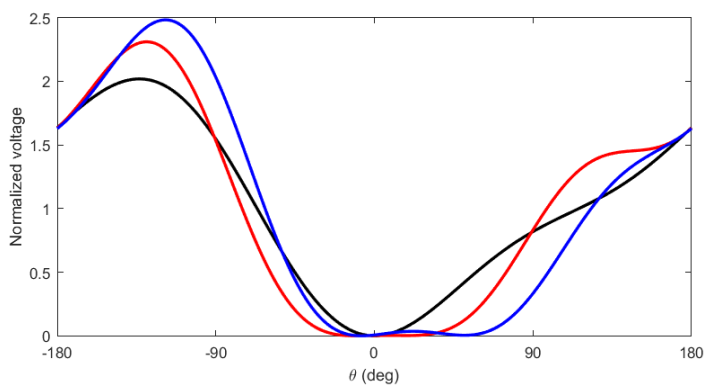

Fig. 9. Normalized voltage Third harmonic loads (a) and fundamental clipping contours (b) for 3 different cases, fixing $Z_{2}=(1+j) R_{\mathrm{opt}} / 2 . Z_{3}=0$ (black), $Z_{3}=5 R_{\text {opt }}$ (red), and $Z_{3}=(5+j 5) R_{\text {opt }} / \sqrt{2}$ (blue).

waveform obtained with the maximum output power load. The experimental evaluation of the proposed third harmonic contours is ongoing, and it will allow for the verification of the beneficial effects of third harmonic on a real device.

\section{CONCLUSiON}

A quick computational method for harmonic clipping contours has been demonstrated using a Matlab script. With respect to previously published methods, the present one allows for the insertion of higher harmonics. In particular, the effect of introducing a third harmonic voltage is discussed, showing the potential benefits in terms of output power and efficiency.

\section{ACKNOWLEDGMENT}

This project has received funding from the European Union's Horizon 2020 research and innovation programme under the Marie Skłodowska-Curie grant agreement No. 654987.

\section{REFERENCES}

[1] P. Colantonio, F. Giannini, and E. Limiti, High Efficiency RF and Microwave Solid State Power Amplifiers, ser. Microwave and Optical Engineering. John Wiley \& Sons, 2009.

[2] S. Cripps, RF power amplifiers for wireless communications, ser. Artech House Microwave Library. Artech House, 2006.

[3] S. Cripps, P. Tasker, A. Clarke, J. Lees, and J. Benedikt, "On the Continuity of High Efficiency Modes in Linear RF Power Amplifiers," IEEE Microw. Wireless Compon. Lett., vol. 19, no. 10, pp. 665 - 667, Oct. 2009.

[4] V. Carrubba, A. Clarke, M. Akmal, J. Lees, J. Benedikt, P. Tasker, and S. Cripps, "On the extension of the continuous class-F mode power amplifier," IEEE Trans. Microw. Theory Tech., vol. 59, no. 5, pp. 12941303, May 2011.

[5] S. Rezaei, L. Belostotski, M. Helaoui, and F. Ghannouchi, "Harmonically tuned continuous class-C operation mode for power amplifier applications," Microwave Theory and Techniques, IEEE Transactions on, vol. 62, no. 12, pp. 3017-3027, Dec 2014.

[6] R. Quaglia, V. Camarchia, M. Pirola, J. Rubio, and G. Ghione, "Linear GaN MMIC Combined Power Amplifiers for 7-GHz Microwave Backhaul," IEEE Trans. Microw. Theory Tech., vol. 62, no. 11, pp. 2700-2710, Nov 2014.

[7] T. Canning, P. Tasker, and S. Cripps, "Continuous mode power amplifier design using harmonic clipping contours: Theory and practice," IEEE Trans. Microw. Theory Tech., vol. 62, no. 1, pp. 100-110, Jan 2014.

[8] C. Friesicke, R. Quay, and A. Jacob, "The resistive-reactive class-J power amplifier mode," IEEE Microw. Wireless Compon. Lett., vol. 25, no. 10, pp. 666-668, Oct 2015. 